ESAIM: PS

February 2007, Vol. 11, p. 35-39

DOI: $10.1051 / \mathrm{ps}: 2007004$
ESAIM: Probability and Statistics

www.edpsciences.org/ps

\title{
CONSISTENT PRICE SYSTEMS FOR SUBFILTRATIONS ${ }^{*, * *}$
}

\author{
Andrea Gombani ${ }^{1}$, Stefan Jaschke ${ }^{2}$ And Wolfgang Runggaldier ${ }^{3}$
}

\begin{abstract}
Asymmetric or partial information in financial markets may be represented by different filtrations. We consider the case of a larger filtration $\mathcal{F}-$ the natural filtration of the "model world" and a subfiltration $\hat{\mathcal{F}}$ that represents the information available to an agent in the "real world". Given a price system on the larger filtration that is represented by a martingale measure $Q$ and an associated numeraire $S$, we show that there is a canonical and nontrivial numeraire $\hat{S}$ such that the price system generated by $(\hat{S}, Q, \hat{\mathcal{F}})$ is consistent, in a sense to be made precise, with the price system generated by $(S, Q, \mathcal{F})$.
\end{abstract}

Mathematics Subject Classification. 91B24, 93E11.

Invited paper accepted September 2005.

\section{INTRODUCTION}

We consider here a general setting with partial or noisy information, where the larger filtration $\mathcal{F}$ denotes the natural filtration of the "model world" and the sub-filtration $\hat{\mathcal{F}}$ represents the information available to an agent in the "real world". An example is the bond market, where in some models one assumes that the uncountably many bonds of all maturities are observed and traded, whereas in practice only a finite number of them is accessible in the market (partial information). In some other models one assumes that all bond prices or swap rates can be explained by a low-dimensional latent process. Since the whole set of observed prices cannot be fitted by the parsimonious model without error, the difference between model and real prices needs to be interpreted as unobserved noise (noisy information).

Given a "model world" filtration $\mathcal{F}$ and a "real world" sub-filtration $\hat{\mathcal{F}}$, we consider models that are arbitragefree in the "model filtration" $\mathcal{F}$, i.e., they admit different martingale measures $Q$ and corresponding $\mathcal{F}$-adapted numeraires $S$ (the concept of numeraire and its properties have been studied in detail by [6]; see also the exposition of the subject by [3]). It is easy to see that, if the numeraire $S$ of $Q$ is $\hat{\mathcal{F}}$-adapted, then $Q$ is a martingale measure also in $\hat{\mathcal{F}}$ for the same numeraire $S$. If $Q^{0}$ and $Q^{1}$ are two martingale measures in $\mathcal{F}$ with numeraires $S^{0}$ and $S^{1}$, respectively, that lead to the same pricing system, then it is also rather straightforward to

Keywords and phrases. Consistent price systems, partial information, numeraire, filtering

* Dedicated to Professor Nicole El Karoui on occasion of her 60th birthday.

** We wish to thank Professor Tomas Björk for various comments and Professor Christophe Stricker for having suggested the present simpler version of the proof of Proposition 3.1.

1 LADSEB-CNR, Corso Stati Uniti 4, 35020 Padova, Italy; gombani@isib.cnr.it

2 BaFin, Graurheindorfer Str. 108, 53117 Bonn, Germany; stefan@jaschke-net.de

3 Dipartimento Matematica Pura ed Applicata, Universitá di Padova, Via Trieste 63, 35121 Padova, Italy; runggal@math.unipd.it

(c) EDP Sciences, SMAI 2007 
see that, if $S^{0}$ and $S^{1}$ are both adapted to the smaller filtration $\hat{\mathcal{F}}$, the two measures $Q^{0}$ and $Q^{1}$ are martingale measures also in $\hat{\mathcal{F}}$ and the triples $\left(S^{0}, Q^{0}, \hat{\mathcal{F}}\right)$ and $\left(S^{1}, Q^{1}, \hat{\mathcal{F}}\right)$ lead to the same pricing system as well. What is not obvious, though, is what happens if the natural numeraire in the model world is not traded nor observed. The answer constitutes the main result of the paper and is stated in Proposition 3.1, where we show that any given numeraire $S$ for the larger filtration $\mathcal{F}$ will generate a canonical and nontrivial numeraire $\hat{S}$ for the smaller filtration $\hat{\mathcal{F}}$, such that the price system generated by $(\hat{S}, Q, \hat{\mathcal{F}})$ is consistent with the price system generated by $(S, Q, \mathcal{F})$.

This main result has been applied to the case of bond markets in [7]. This application also leads to interesting representations of the arbitrage-free bond prices in the smaller filtration, i.e., the arbitrage-free prices of bonds of any maturity, traded or not, that are consistent with the information corresponding to the smaller filtration. In [7] it is furthermore shown how, for exponentially affine term structure models, these prices can be explicitly computed with the use of the Kalman filter.

\section{Consistent PRICE Systems}

Let $L_{t}$ denote the set of traded time- $t$ claims $(0<t \leq T)$. In what follows we shall restrict ourselves to frictionless markets so that $L_{t}$ will be a linear space for $0<t \leq T$.

Definition 2.1. A linear price system $\left\{\Pi_{t, T}, L_{T}\right\}_{t \leq T}$ is a family of linear mappings $\Pi_{t, T}: L_{T} \rightarrow L_{t}$ with the properties

(1) $\Pi_{t_{1}, t_{2}} \circ \Pi_{t_{2}, t_{3}}=\Pi_{t_{1}, t_{3}}$ for all $t_{1} \leq t_{2} \leq t_{3}$ and

(2) $\Pi_{t, t}=i d$.

Think of a price system as the collection of all offers that a market maker makes or all contracts that are liquidly traded on a specific exchange. $\Pi_{t, T}(X)$ is the price at which the time- $T$ claim can be bought and sold at time $t$. Note that this assumes uninterrupted pricing: if a time- $T$ claim is priced at all, it is priced at all prior times $t \leq T$.

Given a triplet $(S, Q, \mathcal{F})$ of a probability measure $Q$, a filtration $\mathcal{F}$ and a positive, $\mathcal{F}$-adapted process $S$, which is bounded away from zero and which for later use we shall assume to be right continuous, the present value equation

$$
\Pi_{t, T}(X):=S_{t} E^{Q}\left[X / S_{T} \mid \mathcal{F}_{t}\right], \quad X \in L_{T}=L^{\infty}\left(\mathcal{F}_{T}\right)
$$

obviously defines a linear price system. To avoid technicalities, in Definition 2.1 we do not require the mappings $\Pi_{t, T}$ to have additional (e.g., continuity) properties, which would allow to show that every "arbitrage-free" price system can be represented in the form (1). Several pairs of topological conditions and notions of noarbitrage/no-free-lunch exist [5] but they are not needed here.

If the domains $L_{t}$ of a price system $\left\{\Pi_{t, T}\right\}$ are defined by a filtration $\mathcal{F}=\left\{\mathcal{F}_{t}\right\}$ as $L_{t}=L^{\infty}\left(\mathcal{F}_{t}\right)$, then we will call $\left\{\Pi_{t, T}\right\}$ "a price system for the filtration $\mathcal{F}$ ". Note that this implies a form of completeness of this price system. Every time- $T$ claim that is "observed" in $\mathcal{F}_{T}$ is priced by $\Pi_{t, T}$ for all prior times $t \leq T$. Specifically, all default-free zero-bonds are priced by $\Pi_{t, T}(1)$.

Definition 2.2. Call two price systems $\left\{\Pi_{t, T}^{1}, L_{T}^{1}\right\}$ and $\left\{\Pi_{t, T}^{2}, L_{T}^{2}\right\}$ consistent, if they agree on the common subspaces: $\Pi_{t, T}^{1}(X)=\Pi_{t, T}^{2}(X)$ for all $X \in L_{T}^{1} \cap L_{T}^{2}$.

In the above interpretation, consistency between two market makers (exchanges) means that an outsider cannot arbitrage between the market makers (exchanges). Another interpretation of consistency is the following. Given a vector $\left(S^{1}, \ldots, S^{n}\right)$ of security prices, the mappings $S_{T}^{i} \mapsto S_{t}^{i}$ define a price system on the spaces spanned by the price vectors $\left(L_{T}:=\operatorname{span}\left(S_{T}^{1}, \ldots, S_{T}^{n}\right)\right)$. Such a price system is obviously consistent with the price system generated by a triplet $(S, Q, \mathcal{F})$ if and only if $Q$ is a martingale measure for the numeraire $S$, i.e., the discounted price processes $S_{t}^{i} / S_{t}$ are $(Q, \mathcal{F})$-martingales. 
Setting. In the rest of this section, we shall consider the situation where we have a "model" price system $\left\{\Pi_{t, T}, L^{\infty}\left(\mathcal{F}_{T}\right)\right\}$ for a larger filtration $\mathcal{F}$ and a "real world" price system $\left\{\hat{\Pi}_{t, T}, \hat{L}_{T}\right\}$ with $\hat{L}_{T} \subseteq L^{\infty}\left(\hat{\mathcal{F}}_{T}\right) \subseteq$ $L^{\infty}\left(\mathcal{F}_{T}\right)$ for some sub-filtration $\hat{\mathcal{F}}$ of $\mathcal{F}$.

This may arise in the case of

noisy information: real world prices may not fit to parsimonious, low-dimensional representations (of forward curves or volatility surfaces), so the existence of unobserved noise may be needed to match model and reality;

partial information: the power of mathematical analysis is used to build "nice" models for a continuum of prices, while only finitely many securities (e.g., points on forward curves or volatility surfaces) are liquidly traded;

incomplete markets: not all observable time- $T$ claims need to be traded $\left(\hat{L}_{T} \neq L^{\infty}\left(\hat{\mathcal{F}}_{T}\right)\right)$.

Proposition 2.3. Consider a price system $\left\{\Pi_{t, T}, L^{\infty}\left(\mathcal{F}_{T}\right)\right\}$ for the filtration $\mathcal{F}$, a sub-filtration $\hat{\mathcal{F}}$ of $\mathcal{F}$, and a set of linear spaces $\hat{L}_{T} \subseteq L^{\infty}\left(\hat{\mathcal{F}}_{T}\right)$. The set $\left\{\hat{L}_{T}\right\}$ can carry a price system that is consistent with $\left\{\Pi_{t, T}, L^{\infty}\left(\mathcal{F}_{T}\right)\right\}$ if and only if

$$
X \in \hat{L}_{T} \Longrightarrow \Pi_{t, T}(X) \in \hat{L}_{t}, \quad t \leq T
$$

Proof. It is clear that the only candidate for a consistent price system on the sub-spaces $\hat{L}_{T}$ is the restriction of each of the mappings $\Pi_{t, T}$ to $\hat{L}_{T}$. Denote this restricted price system ${ }^{1}$ by $\left\{\hat{\Pi}_{t, T}\right\}$. The condition (2) of Definition 2.1 is automatically fulfilled by the restricted price system. What remains to be shown is first that the restriction $\hat{\Pi}_{t, T}$ of $\Pi_{t, T}$ to $\hat{L}_{T}$ maps into $\hat{L}_{t}$. But this is just a reformulation of condition (2). Second, condition (1) of Definition 2.1 needs to be checked. From $\Pi_{t_{1}, t_{2}} \circ \Pi_{t_{2}, t_{3}}=\Pi_{t_{1}, t_{3}}$ follows $\Pi_{t_{1}, t_{2}} \circ \hat{\Pi}_{t_{2}, t_{3}}=\hat{\Pi}_{t_{1}, t_{3}}$ by restricting both sides to $\hat{L}_{t_{3}}$. Condition (2) ensures $\Pi_{t_{1}, t_{2}} \circ \hat{\Pi}_{t_{2}, t_{3}}=\hat{\Pi}_{t_{1}, t_{2}} \circ \hat{\Pi}_{t_{2}, t_{3}}$.

If condition (2) is not fulfilled, then the image of $\hat{L}_{T}$ under the mapping $\Pi_{t, T}$ is not contained in $\hat{L}_{t}$.

Note that condition (2) implies "uninterrupted pricing" again. Whenever a time- $T$ claim is (in the "real world") priced at all, then it is priced for all prior times $t \leq T$.

Proposition 2.4. Consider a price system $\left\{\Pi_{t, T}, L^{\infty}\left(\mathcal{F}_{T}\right)\right\}$ for the filtration $\mathcal{F}$, a sub-filtration $\hat{\mathcal{F}}$ of $\mathcal{F}$, and a set of linear spaces $\hat{L}_{T} \subseteq L^{\infty}\left(\hat{\mathcal{F}}_{T}\right)$ that can carry a price system that is consistent with $\left\{\Pi_{t, T}, L^{\infty}\left(\mathcal{F}_{T}\right)\right\}$. If $\left\{\Pi_{t, T}\right\}$ is generated by the triplet $(S, Q, \mathcal{F})$ as in $(1)$ and $S$ is $\hat{\mathcal{F}}$-adapted, then the "filtered price system"

$$
\hat{\Pi}_{t, T}(X):=S_{t} E^{Q}\left[X / S_{T} \mid \hat{\mathcal{F}}_{t}\right], \quad \forall X \in \hat{L}_{T}
$$

equals the unique price system on the sub-spaces $\hat{L}_{T}$ that is consistent with $\left\{\Pi_{t, T}, L^{\infty}\left(\mathcal{F}_{T}\right)\right\}$.

Proof. Since $S$ is $\hat{\mathcal{F}}$-adapted,

$$
\hat{\Pi}_{t, T}(X)=E^{Q}\left[S_{t} X / S_{T} \mid \hat{\mathcal{F}}_{t}\right]=E^{Q}\left[\Pi_{t, T}(X) \mid \hat{\mathcal{F}}_{t}\right]
$$

which equals $\Pi_{t, T}(X)$ for all $X \in \hat{L}_{T}$ because of condition (2).

Remark 2.5. Note that $\hat{\Pi}_{t, T}$ in (3) can be defined for larger domains than $\hat{L}_{T}$, but only its restriction to a family of spaces $\left\{\hat{L}_{T}\right\}$ with property (2) is consistent with the "model" price system and, on those subspaces $\hat{L}_{T}$, it is uniquely determined.

\footnotetext{
${ }^{1}$ Note that there might be different triplets $(S, Q, \hat{\mathcal{F}})$ that generate via $(1)$ the same mappings $\hat{\Pi}_{t, T}$ when restricted to $\hat{L}_{T}$. The restricted price system is unique as a family of mappings in the sense of Definition 2.1.
} 
Notice, furthermore, that the context of Proposition 2.4 gives an example where the so-called "Hypothesis H" holds (see [4]; see also the discussion around formula (1) in [1]), namely in the sense that the discounted prices $\hat{\Pi}_{t, T}$, which are martingales in the smaller filtration $\hat{\mathcal{F}}_{t}$, remain martingales also in the larger filtration $\mathcal{F}_{t}$.

\section{MAIN RESUlT}

A consequence of Proposition 2.4 is that the filtering (3) is independent of which pair $(S, Q)$ is used to represent the "model price system" $\left\{\Pi_{t, T}\right\}$. In the pricing of interest rate derivatives it is customary to switch the representation $(S, Q)$ as is most appropriate $[2,3,6]$.

The crucial question is now how to "filter", if the natural numeraire in the model world is not or only noisily observed in the real world, i.e., $S$ is not $\hat{\mathcal{F}}$-adapted. Asked differently, does there exist - in the setting of Proposition 2.4 but without the assumption that $S$ is $\hat{\mathcal{F}}$-adapted - a process $\hat{S}$ such that the unique consistent price system on the sub-spaces is generated by the triplet $(Q, \hat{S}, \hat{\mathcal{F}})$ ?

Proposition 3.1. Consider a price system $\left\{\Pi_{t, T}\right\}$ for the filtration $\mathcal{F}$, a sub-filtration $\hat{\mathcal{F}}$ of $\mathcal{F}$, and a set of linear spaces $\hat{L}_{T} \subseteq L^{\infty}\left(\hat{\mathcal{F}}_{T}\right)$ that can carry a price system that is consistent with $\left\{\Pi_{t, T}\right\}$. If $\left\{\Pi_{t, T}\right\}$ is generated by the triplet $(S, Q, \mathcal{F})$ as in $(1)$, then the unique consistent price system on the sub-filtration is given by

$$
\begin{aligned}
\hat{\Pi}_{t, T}(X) & :=\hat{S}_{t} E^{Q}\left[X / \hat{S}_{T} \mid \hat{\mathcal{F}}_{t}\right] \\
\hat{S}_{t} & :=1 / E^{Q}\left[1 / S_{t} \mid \hat{\mathcal{F}}_{t}\right],
\end{aligned}
$$

where, in order to guarantee right continuity of $\hat{S}_{t}$ in (5) and thus its uniqueness, we take the optional version of the conditional expectation.

Proof. Fix a contingent claim $X \in \hat{L}_{T}$. Starting from (1), i.e.,

$$
\frac{\Pi_{t, T}(X)}{S_{t}}=E^{Q}\left[\frac{X}{S_{T}} \mid \mathcal{F}_{t}\right]
$$

we get on the one hand

$$
E^{Q}\left[\frac{\Pi_{t, T}(X)}{S_{t}} \mid \hat{\mathcal{F}}_{t}\right]=E^{Q}\left[\frac{X}{S_{T}} \mid \hat{\mathcal{F}}_{t}\right]=E^{Q}\left\{X E^{Q}\left[\frac{1}{S_{T}} \mid \hat{\mathcal{F}}_{T}\right] \mid \hat{\mathcal{F}}_{t}\right\}
$$

On the other hand

$$
E^{Q}\left[\frac{\Pi_{t, T}(X)}{S_{t}} \mid \hat{\mathcal{F}}_{t}\right]=\Pi_{t, T}(X) E^{Q}\left[\frac{1}{S_{t}} \mid \hat{\mathcal{F}}_{t}\right]
$$

where we have used the fact that the consistency condition $(2)$ implies $\Pi_{t, T}(X) \in \hat{L}_{t}$. From $(6)$ and $(7)$ we then have

$$
\Pi_{t, T}(X)=\frac{E^{Q}\left\{X E^{Q}\left[\frac{1}{S_{T}} \mid \hat{\mathcal{F}}_{T}\right] \mid \hat{\mathcal{F}}_{t}\right\}}{E^{Q}\left[\frac{1}{S_{t}} \mid \hat{\mathcal{F}}_{t}\right]} .
$$

Remark 3.2. The expression for the numeraire $\hat{S}_{t}$ in (5) becomes more intuitive by recalling that numeraires appear in the denominator and by rewriting, consequently, relation (5) as $1 / \hat{S}_{t}=E^{Q}\left\{1 / S_{t} \mid \hat{\mathcal{F}}_{t}\right\}$. 


\section{REFERENCES}

[1] M. Atlan, H. Geman, D.B. Madan and M. Yor, Correlation and the pricing of risks. Prépublications du Laboratoire de Probabilités et Modèles Aléatoires (2004), No. 877.

[2] T. Björk, Interest rate theory, in Financial Mathematics, W.J. Runggaldier Ed., Lecture Notes in Mathematics, Springer, Berlin (1997) 53-122.

[3] T. Björk, Arbitrage Theory in Continuous Time. Oxford University Press (1998/2004).

[4] P. Brémaud and M. Yor, Changes of Filtrations and of Probability Measures. Z. Wahrscheinlichkeitstheorie verw. Gebiete 45 (1978) 269-295.

[5] F. Delbaen and W. Schachermayer, Non-arbitrage and the fundamental theorem of asset pricing: Summary of main results. http://www.math.ethz.ch/ delbaen/. Proceedings of Symposia in Applied Mathematics of the AMS (1997).

[6] H. Geman, N. El Karoui and J.-C. Rochet, Changes of numéraire, changes of probability measure and option pricing. J. Appl. Probab. 32 (1995) 443-458.

[7] A. Gombani, S. Jaschke and W. Runggaldier, A filtered no arbitrage model for term structures from noisy data. Stochastic Processes Appl. 115 (2005) 381-400. 